Original Article

\title{
Randomized controlled comparative study on effect of training to improve lower limb motor paralysis in convalescent patients with post-stroke hemiplegia
}

\author{
Kenji Kawakami, RPT ${ }^{1)^{*}}$, Hiroyuki Miyasaka, OTR ${ }^{1,2)}$, Sayaka Nonoyama, RPT ${ }^{3)}$, \\ Kazuya Hayashi, RPT ${ }^{4}$, Yusuke Tonogai, RPT ${ }^{1)}$, Genichi Tanino, RPT ${ }^{1,2}$, \\ Yosuke Wada, RPT ${ }^{5}$, Akinisa Narukawa, RPT ${ }^{6}$, Yuko OKuyama, RPT ${ }^{1}$, \\ Yutaka TOMita, RE ${ }^{2}$, Shigeru SONODA, MD $^{1,2)}$ \\ 1) Fujita Health University Nanakuri Sanatorium: 424-1 Ohdori, Tsu-shi, Mie 514-1295, Japan \\ 2) Division of Rehabilitation, Fujita Memorial Nanakuri Institute, Japan \\ 3) Fujita Health University Core Center of The Regional Comprehensive Care, Japan \\ 4) Fujita Health University Hospital, Japan \\ 5) Tsujimura Surgical Hospital, Japan \\ 6) Zenjyokai Rehabilitation Hospital, Japan
}

\begin{abstract}
Purpose] The motor paralysis-improving effect on the hemiplegic lower limb was compared among mirror therapy, integrated volitional-control electrical stimulation, therapeutic electrical stimulation, repetitive facilitative exercises, and the standard training method in post-stroke hemiplegia patients. [Subjects and Methods] Eighty one stroke patients admitted to a convalescent rehabilitation ward were randomly allocated to the above 5 treatment groups. Each patient performed functional training of the paralytic lower limb for 20 minutes a day for 4 weeks, and changes in the lower limb function were investigated using the Stroke Impairment Assessment Set. [Results] The hip and knee joint functions did not significantly improve in the standard training control group, but significant improvements were observed after 4 weeks in the other intervention groups. Significant improvement was noted in the ankle joint function in all groups. [Conclusion] Although the results were influenced by spontaneous recovery and the standard training in the control group, the hip and knee joints were more markedly improved by the interventions in the other 4 groups of patients with moderate paralysis, compared to the control group.

Key words: Stroke, Motor paralysis, Intervention
\end{abstract}

(This article was submitted May 11, 2015, and was accepted Jun. 18, 2015)

\section{INTRODUCTION}

Regarding rehabilitation of stroke patients, many studies reported improvement of activities of daily living (ADL) and gait $^{1-3)}$

ADL and the walking ability are considered to be correlated with motor paralysis ${ }^{4}$, and the improvement of motor paralysis may lead to improvements of ADL and the walking ability.

Pollock et al. ${ }^{5)}$ stated in a systematic review of therapy for lower limb paralysis that an intervention combining different approaches, such as a combination of gait training and muscle-strengthening exercise, is more effective than waiting for spontaneous recovery without training. However, they concluded that there is no evidence to identify a training

*Corresponding author. Kenji Kawakami (E-mail: kawakamikenji07n@yahoo.co.jp)

(C)2015 The Society of Physical Therapy Science. Published by IPEC Inc. This is an open-access article distributed under the terms of the Creative Commons Attribution Non-Commercial No Derivatives (by-ncnd) License $<$ http://creativecommons.org/licenses/by-nc-nd/3.0/>. method as effective among the various methods available.

Regarding rehabilitation of stroke patients with lower limb motor paralysis, Sutbeyaz et al. ${ }^{6}$ reported that mirror therapy of the lower limbs improved motor paralysis and the walking ability on the paralytic side in stroke patients, Merletti et al. ${ }^{7}$ ) reported that therapeutic electrical stimulation of the ankle joint flexors improved motor paralysis of the paralytic ankle joint in stroke patients, and Kawahira et al. ${ }^{8)}$ reported that a repetitive facilitative exercise program of the hip, knee, and ankle joints performed 100 times a day improved motor paralysis of the lower limb and increased the muscle force on bending and extending the knee joint in stroke patients.

Most previous studies compared the intervention and control groups, as described below, and did not investigate which of the various training methods is effective.

In this study, to clarify differences in the motor paralysisimproving effect, the following 4 interventions recently reported to be effective were compared with regard to treatment of the paralytic lower limb in post-stroke hemiplegia patients, setting a control group (Con) treated with the standard training method: mirror therapy $(\mathrm{MT})^{6,9)}$, integrated volitional-control electrical stimulation (IVES) ${ }^{10)}$, therapeu- 
tic electrical stimulation (TES $)^{7)}$, and repetitive facilitative exercises (RFEs $)^{8)}$.

\section{SUBJECTS AND METHODS}

The subjects were 81 patients with hemiplegia following initial supratentorial stroke admitted to a convalescent rehabilitation ward of our hospital between September 2009 and July 2011 who did not meet the exclusion criteria described below. Attending physicians explained the study to all patients meeting these conditions and their families, and obtained consent. This study was performed after approval (No. 46) by the Ethics Committee of our hospital.

The exclusion criteria were: the time to admission from the onset is within 14 days, difficult communication due to severe cognitive disorder, comorbidity index of 4 or high$\mathrm{er}^{11)}$, necessity of high-level consideration and caution for rehabilitation, and scores of Hip-flexion, Knee-extension, and Foot-pat items of the Stroke Impairment Assessment Set $\left(\right.$ SIAS) ${ }^{12)}$ lower than 2.

For the study design, a randomized controlled study by random allocation was adopted. In randomization, the patients were allocated in the order of entry using a table of random numbers. The training methods of the 5 groups were MT, IVES, TES, RFEs, and standard training (control group: Con), respectively. The standard training was comprised of range of motion and ADL training, and the training methods received by the other 4 groups were not employed. This study attached greater importance to the balance with ADL training. Training that can be completed within 20 minutes a day and has been reported to be effective by studies on motor paralysis and randomized controlled trials (RCT) involving hemiplegia patients was adopted. The time for physical, occupational, and speech therapies was set to one hour each, and the assigned training method was performed during physical therapy to standardize the training time. When no speech therapy was prescribed, the conventional physical or occupational therapy was performed.

In MT, a self-made device was placed as shown in Fig. 1, and the patients performed 3 types of exercise for 20 minutes (dorsiflexion of the ankle joint, stepping over, and abduction/ adduction of the hip joint) while reflecting the non-paralytic lower limb in the mirror. On ankle dorsiflexion, the patients were instructed to perform dorsiflexion of the non-paralytic ankle and simultaneously move the paralytic side, during which the heel of the paralytic side was pressed to fix the leg position. The patients performed 4 sets of 50 repetitions of this motion at a comfortable rhythm. On stepping over, the patients stepped on and down from a wooden block in front (the square timber on which the mirror was placed) with the non-paralytic leg. They were instructed to imagine moving the paralytic side, not actually try to move it, and performed 2 sets of 50 repetitions of this motion at a comfortable rhythm. Abduction/adduction of the hip joint was performed on the non-paralytic side, and the patients were instructed to simultaneously imagine moving the paralytic side. Two sets of 50 repetitions of this motion were performed.

In IVES, dorsiflexion of the ankle joint and extension of the knee joint on the paralytic side were performed for 10 minutes, respectively, while sitting on a chair (Fig. 2), and

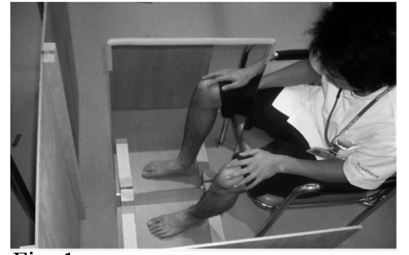

Fig. 1

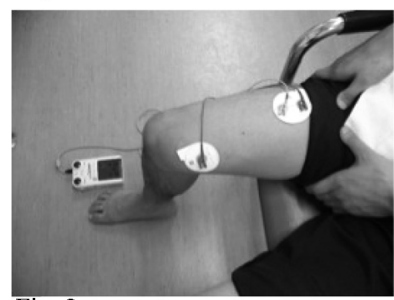

Fig. 3.

Fig. 1. Training scene of MT

Fig. 2. Training scene of IVES

Fig. 3. Training scene of TES

Fig. 4. Training scene of RFEs

electrical stimulation (low frequency) with $50 \mu$ s pulse width, $20 \mathrm{~Hz}$ frequency bidirectional square waves was applied at an intensity proportional to the voluntary myoelectric activity level on the paralytic side using the power assist mode of the Power Assist Stimulator system (OG Giken, Okayama, Japan). All patients performed the exercise at a comfortable timing. When movement paused and no voluntary electromyogram was detected, electrical stimulation at the muscle contractile threshold was applied to facilitate voluntary movement. The electrode was attached to the anterior tibial muscle on ankle dorsiflexion, and rectus femoris and medial great muscles on knee extension.

In TES, electrical stimulation with $50 \mu$ s pulse width, 20 $\mathrm{Hz}$ frequency bidirectional square waves was applied at the maximum acceptable intensity during 10 minutes each of paralytic ankle dorsiflexion and knee extension exercises using the normal mode of the Power Assist Stimulator system (Fig. 3). A 5-second cycle comprised of 2 seconds of turning on and 3 seconds of turning off electricity was repeated, and the patients performed voluntary movement when the electricity was turned on. The electrodes were attached to the same sites as in IVES.

RFEs are treatment aiming at reconstructing and strengthening the motor descending tract through repeating intentional movements of patients using the stretch reflex. The patients performed ankle dorsiflexion 100 or more times during a 10-minute period in a supine position using manual tapping stimulation (Fig. 4). In addition, they performed hip flexion-extension exercise, abduction-adduction exercise, extension/abduction-flexion/adduction exercise, and hip extension/abduction/retention of external rotation/ knee extension-hip flexion/adduction/external rotation/knee flexion exercise in the remaining 10 minutes.

In Con (Standard training), only range of passive motion training and active assistive movement other than the above 4 training methods were performed as functional training. In addition, especial physical agents and facilitation techniques were not used. As for motion exercise, physical therapy 
Table 1. Characteristics of each training groups

\begin{tabular}{lccccccc}
\hline & $\begin{array}{c}\text { Number of } \\
\text { patients }\end{array}$ & $\begin{array}{c}\text { Age } \\
\text { (years) }\end{array}$ & $\begin{array}{c}\text { Time after } \\
\text { onset } \\
\text { (days) }\end{array}$ & $\begin{array}{c}\text { Gender } \\
\text { (Male, Female) }\end{array}$ & $\begin{array}{c}\text { Paralytic side } \\
\text { (Right, Left) }\end{array}$ & $\begin{array}{c}\text { Diagnosis } \\
\text { (Hemorrhage, } \\
\text { Infarction) }\end{array}$ & $\begin{array}{c}\text { Mean number } \\
\text { of physical } \\
\text { therapy units }\end{array}$ \\
\hline MT & 16 & $61.6 \pm 12.7$ & $37.9 \pm 11.8$ & 11,5 & 6,10 & 10,6 & $3.5 \pm 0.7$ \\
IVES & 19 & $66.6 \pm 14.9$ & $33.5 \pm 13.3$ & 12,7 & 10,9 & 10,9 & $3.7 \pm 0.8$ \\
TES & 15 & $65.4 \pm 13.3$ & $32.7 \pm 9.6$ & 11,4 & 8,7 & 8,7 & $3.8 \pm 0.9$ \\
RFEs & 9 & $60.1 \pm 12.7$ & $33.2 \pm 9.7$ & 4,5 & 3,6 & 7,2 & $3.5 \pm 0.8$ \\
Con & 8 & $65.6 \pm 15.9$ & $38.9 \pm 14.8$ & 5,3 & 5,3 & 4,4 & $3.1 \pm 0.4$ \\
\hline
\end{tabular}

Data are numbers, mean \pm SD

n.s.

Table 2. Within-group comparison of the Hip-flexion, Knee-extension, and Foot-pat scores

\begin{tabular}{lcccccc}
\hline & \multicolumn{2}{c}{ Hip-Flexion } & \multicolumn{2}{c}{ Knee-Extension } & \multicolumn{2}{c}{ Foot-Pat } \\
& admission & 4-week & admission & 4-week & admission & 4-week \\
\hline MT & $3(3.3)$ & $4(3.9)^{* *}$ & $3(3.3)$ & $4(3.9)^{* *}$ & $3(3.1)$ & $4(3.8)^{* *}$ \\
IVES & $3(3.2)$ & $4(3.6)^{*}$ & $3(3.2)$ & $4(3.8)^{* *}$ & $3(3.0)$ & $4(3.6)^{* *}$ \\
TES & $3(3.0)$ & $4(3.7)^{* *}$ & $3(3.1)$ & $4(3.7)^{* *}$ & $3(3.0)$ & $4(3.6)^{*}$ \\
RFEs & $4(3.7)$ & $4(4.2)^{*}$ & $4(3.6)$ & $4(4.1)^{*}$ & $4(3.8)$ & $4(4.2)^{*}$ \\
Con & $4(3.9)$ & $5(4.3)$ & $4(3.6)$ & $4(3.9)$ & $3(3.2)$ & $4(3.8)^{*}$ \\
\hline \multicolumn{2}{l}{ Data are medians (mean) } \\
*Wilcoxon signed-ranks test $\mathrm{p}<0.05$ & & & & \\
**Wilcoxon signed-ranks test $\mathrm{p}<0.01$ & & & &
\end{tabular}

centering on the standard gait, standing, balance and ADL training were performed. These training ratio has not been determined.

The SIAS Hip-Flexion, Knee-Extension, and Foot-Pat items were evaluated on admission and after 4 weeks by the attending physicians and physical therapists. Patient information, such as the age, gender, diagnosis, paralytic side, and time after onset, were investigated in the medical records, and the mean number of physical therapy units performed per day in the period from admission to 4 weeks was calculated.

Changes in the SIAS Hip-Flexion, Knee-Extension, and Foot-Pat scores over the 4 weeks from admission were analyzed using the Wilcoxon signed-ranks test. The gains were calculated by subtracting the SIAS Hip-Flexion, KneeExtension, and Foot-Pat scores on admission from those at 4 weeks. These gains, the age, time after onset, and mean number of physical therapy units performed were compared using the Kruskal-Wallis test, and the gender ratio and rates of the paralytic side and diagnosis were compared using the $\chi^{2}$ test.

For statistical analysis, SPSS version 16.0 for Mac (IBM Japan Ltd., Tokyo, Japan) was used, and the significance level was set at less than $5 \%$.

\section{RESULTS}

After the initiation of training, 3, 6, 2, 2, and 1 patient dropped out from the MT, IVES, TES, RFEs, and control groups, respectively, due to a poor condition, an unwillingness to participate, and difficulty in training. The profiles of the analysis set excluding these subjects are shown in
Table 3. Inter-group comparison of the Hip-Flexion, KneeExtension, and Foot-Pat scores

\begin{tabular}{lccl}
\hline & $\begin{array}{c}\text { Hip- } \\
\text { Flexion gain }\end{array}$ & $\begin{array}{c}\text { Knee- } \\
\text { Extension gain }\end{array}$ & $\begin{array}{c}\text { Foot- } \\
\text { Pat gain }\end{array}$ \\
\hline MT & $1(0.6)$ & $1(0.6)$ & $1(0.6)$ \\
IVES & $0(0.4)$ & $1(0.6)$ & $1(0.7)$ \\
TES & $1(0.7)$ & $1(0.6)$ & $0(0.6)$ \\
RFEs & $0(0.6)$ & $1(0.6)$ & $0(0.4)$ \\
Con & $0(0.4)$ & $1(0.3)$ & $1(0.6)$ \\
\hline
\end{tabular}

Data are medians (mean)

n.s.

Table 1. No significant difference was noted in the age, time after onset, gender, paralytic side, diagnosis, or the mean number of physical therapy units among the groups.

The Hip-Flexion and Knee-Extension scores significantly improved over the 4 -week period after admission in the 4 intervention groups excluding the control group (Table 2). The Foot-Pat score significantly improved over the 4-week period in all groups (Table 2).

No significant difference was noted in the gain of any item among the groups (Table 3 ).

\section{DISCUSSION}

In previous meta-analyses on improvement of the motor function of the hemiplegic lower limb, significant improvement of ADL and functional disorder with an increase in the training time was noted, but the training method and level of intervention required have not been clarified ${ }^{5,13,14)}$. In our 
study, the duration of physical therapy was the same in all groups, suggesting that factors other than the training time influenced the improvement of paralysis. Since the interventions were designed based on therapies reported to be effective $^{6-8,15)}$, patients with a score of 2 or higher in each of Hip-Flexion, Knee-Extension, Foot-Pat were selected. Thus, no patient with severe paralysis was included.

Hip-Flexion and Knee-Extension significantly improved over the period from admission to 4 weeks in all 4 intervention groups excluding the control group. Improvement of lower limb paralysis by MT, TES, and RFEs compared to the control group has been reported ${ }^{6-8)}$. Significant improvement was also noted in our study, clarifying that TES, MT, and RFEs improve lower limb paralysis. For IVES, no study on improvement of lower limb paralysis has been performed, and its effect and application criteria have not been clarified. Barth E et al. ${ }^{15)}$ reported that application of EMG-triggering electrical stimulation during ankle dorsiflexion increased the range of active motion compared to those before intervention in stroke patients, and Miyasaka et al. ${ }^{16)}$ reported that IVES applied to post-stroke hemiplegic upper limbs improved paralysis in patients in whom voluntary contraction was detected. In our study, patients with a detectable voluntary electromyogram were included in the IVES group, suggesting that IVES is effective to improve paralysis in these patients. Since the patients targeted by us are in the convalescent stage, the motor function may readily recover spontaneously, but functional training of the hip and knee joints on the paralytic side may more markedly improve the functions than spontaneous recovery.

On the Foot-Pat test, significant improvement was noted over the period from admission to 4 weeks in all groups, clarifying that there was no efficacy to improve paralysis in the intervention groups. We provide a 3-hour training session per day, 7 days a week, and the frequency and intensity of training are sufficient ${ }^{17)}$. The effect on the ankle joint may have been influenced by the frequency and intensity of the standard training. The effects of the interventions were noted only in the hip and knee joints, and this may have been due to the anatomy of these joints: these attach to larger muscles than those attached by the ankle joint, in which the effect of facilitation may be more easily obtained.

Based on our results, there were no significantly different effects among the 4 methods for the hip and knee joint. However, between our evaluation of the admission and 4 week, there was a significant change in the 4 method groups while there was no significance in the control group. These differences of the course demonstrate the superiority of 4 intervention methods to standard training in control group. For the ankle joint, it may be necessary to develop a training method readily achieving facilitation, and increase the training time for paralysis.

No significant difference was observed among the intervention groups. The interventions were designed to complete in a short time (20 minutes) within the limited training time to fit in the range allowable by the Japanese medical payment system. To obtain clear differences in improvement, it may be necessary to investigate extension of the training time. Moreover, it was unclear whether or not the patients performed voluntary training in addition to therapy by therapists, and the content of voluntary training was also unclear. These may have an influence on improvement of the motor function. It may be necessary to investigate the content of training other than the specified intervention.

We are planning to investigate the selection of functional training corresponding to the severity of paralysis and ratio of training time for paralysis to construct a system to synergistically improve the functions and ADL.

\section{REFERENCES}

1) Iwai $\mathrm{N}$, Aoyagi $\mathrm{Y}$, Tokuhisa $\mathrm{K}$, et al.: The gaps between capability ADL and performance $\mathrm{ADL}$ of stroke patients in a convalescent rehabilitation ward - based on the functional independence measure - . J Phys Ther Sci, 2011, 23: 333-338. [CrossRef]

2) Kwakkel G, van Peppen R, Wagenaar RC, et al.: Effects of augmented exercise therapy time after stroke: a meta-analysis. Stroke, 2004, 35: 2529 2539. [Medline] [CrossRef]

3) Park IM, Lee YS, Moon BM, et al.: A comparison of the effects of overground gait training and treadmill gait training according to stroke patients' gait velocity. J Phys Ther Sci, 2013, 25: 379-382. [CrossRef]

4) Cho KH, Lee JY, Lee KJ, et al.: Factors related to gait function in poststroke patients. J Phys Ther Sci, 2014, 26: 1941-1944. [Medline] [CrossRef]

5) Pollock A, Baer G, Langhorne P, et al.: Physiotherapy treatment approaches for the recovery of postural control and lower limb function following stroke: a systematic review. Clin Rehabil, 2007, 21: 395-410. [Medline] [CrossRef]

6) Sütbeyaz S, Yavuzer G, Sezer N, et al.: Mirror therapy enhances lowerextremity motor recovery and motor functioning after stroke: a randomized controlled trial. Arch Phys Med Rehabil, 2007, 88: 555-559. [Medline] [CrossRef]

7) Merletti R, Zelaschi F, Latella D, et al.: A control study of muscle force recovery in hemiparetic patients during treatment with functional electrical stimulation. Scand J Rehabil Med, 1978, 10: 147-154. [Medline]

8) Kawahira K, Shimodozono M, Ogata A, et al.: Addition of intensive repetition of facilitation exercise to multidisciplinary rehabilitation promotes motor functional recovery of the hemiplegic lower limb. J Rehabil Med, 2004, 36: 159-164. [Medline] [CrossRef]

9) Wada Y, Kondoh I, Sonoda S, et al.: Mirror therapy for severely affected ankle joints of stroke patients. Jpn J Compr Rehabil Sci, 2011, 2: 71-76.

10) Muraoka $Y$ : Development of an EMG recording device from stimulation electrodes for functional electrical stimulation. Front Med Biol Eng, 2002, 11: 323-333. [Medline] [CrossRef]

11) Liu M, Domen K, Chino N: Comorbidity measures for stroke outcome research: a preliminary study. Arch Phys Med Rehabil, 1997, 78: 166-172. [Medline] [CrossRef]

12) Chino N, Sonoda S, Domen K, et al.: Stroke impairment assessment set (SIAS): a new evaluation instrument for stroke patients. Jpn J Rehabil Med, 1994, 31: 119-125. [CrossRef]

13) Kwakkel G, Wagenaar RC, Koelman TW, et al.: Effects of intensity of rehabilitation after stroke. A research synthesis. Stroke, 1997, 28: 15501556. [Medline] [CrossRef]

14) Paci M: Physiotherapy based on the Bobath concept for adults with poststroke hemiplegia: a review of effectiveness studies. J Rehabil Med, 2003, 35: 2-7. [Medline] [CrossRef]

15) Barth E, Herrman V, Levine P, et al.: Low-dose, EMG-triggered electrical stimulation for balance and gait in chronic stroke. Top Stroke Rehabil, 2008, 15: 451-455. [Medline] [CrossRef]

16) Miyasaka H, Ohnishi H, Hieda $\mathrm{C}$, et al.: A study of the training method of sub-acute stroke patients of the upper extremity: decision tree analysis. Jpn J Compr Rehabil Sci, 2014, 5: 117-124.

17) Sonoda $S$, Saitoh E, Nagai $S$, et al.: Full-time integrated treatment program, a new system for stroke rehabilitation in Japan: comparison with conventional rehabilitation. Am J Phys Med Rehabil, 2004, 83: 88-93. [Medline] [CrossRef] 